\title{
Tratamiento de efluentes de lubricadoras y lavadoras de autos mediante un sistema de lodos activados de lechos suspendido a escala de laboratorio.
}

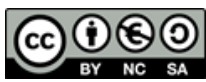

Waterwater treatment from automotive lubricants and washing by system of activated sludges of suspense at laboratory scale

Fabián Morales Fiallos. ${ }^{1}$, Renato Sánchez Proaño. ${ }^{2} \&$ Rodrigo Acosta Lozada. ${ }^{3}$

Recibido: 10-10-2018 / Revisado: 17-11-2018 /Aceptado: 04-12-2018/ Publicado: 05-01-2019

\begin{abstract}
.
DOI: $\underline{\text { https://doi.org/10.33262/cienciadigital.v3i1.278 }}$

Wastewater from car washers and lubricants produces effluents with high concentrations of oils, greases and detergents. A fraction of these pollutants form an emulsion in the water, which when solubilized increase the organic load in parameters such as TOC, COD and $\mathrm{BOD}_{5}$; These emulsified effluents are not separated by primary systems such as grease traps. To evaluate the contamination, a simple sampling was carried out in 61 washing machines and lubricators in the city of Ambato. With these data, a sample representative of $95 \%$ of the total establishments was created in the laboratory; to which the non-emulsified fraction was separated by a primary treatment and the soluble phase was treated with a suspended bed activated sludge reactor. In order to improve the nutritional relationship of the residual water of mineral origin, a suspended bed of sugar cane was placed in the aeration tank.
\end{abstract}

Keywords: Waters, Residuals, Lubricator, Washer, Fats, Emulsified Minerals, Biological Treatment, Fats, Oils.

1 Facultad de Ingeniería Civil y Mecánica, Universidad Técnica de Ambato, Ambato, Ecuador, frmorales@uta.edu.ec

2 Grupo de Investigación en Ciencias Ambientales; Universidad Politécnica Salesiana Ecuador, Sede Quito, rsanchezp@ups.edu.ec

3 Facultad de Ingeniería Civil y Mecánica, Universidad Técnica de Ambato, Ambato, Ecuador, ri.acosta@uta.edu.ec 


\section{Resumen.}

Las aguas residuales procedentes de lavadoras y lubricadoras de automóviles producen efluentes con altas concentraciones de aceites, grasas y detergentes. Una fracción de estos contaminantes forman una emulsión en el agua, que al solubilizarse aumentan la carga orgánica en parámetros como el COT, la DQO y la DBO 5 ; estos efluentes emulsionados no son separados por sistemas primarios como trampas de grasa. Para evaluar la contaminación se procedió a realizar un muestreo simple en 61 lavadoras y lubricadoras de la ciudad de Ambato. Con estos datos se procedió a crear en laboratorio una muestra representativa al 95\% del total de los establecimientos; a la cual se le separó la fracción no emulsionada mediante un tratamiento primario y se trató la fase soluble con un reactor de lodos activados de lecho suspendido. Para mejorar la relación nutricional del agua residual de procedencia mineral se procedió a clocar un lecho suspendido de caña de azúcar en el tanque de aireación

Palabras claves: Aguas, Residuales, Lubricadora, Lavadora, Grasas, Minerales Emulsionadas, Tratamiento Biológico, Grasas, Aceites,

\section{Introducción.}

Las lavadoras y lubricadoras de autos generan efluentes con aceites y grasas; las concentraciones van desde $300 \mathrm{mg} / \mathrm{L}$ hasta $600 \mathrm{mg} / \mathrm{L}$ (Debabrata, 2011). Otros contaminantes como los detergentes se adhieren a los aceites y las grasas no emulsionadas, y al ser estas de una densidad menor al agua se separan en dos fases (Aranberri, Binks, Clint, \& Fletcher, 2010), pudiéndose tratar con sistemas primarios (Techobanglous, 1995).

Los aceites minerales procedentes del sistema de las lavadoras de autos, no tienen buenas propiedades biodegradables (Jiménez, 2012), algo que se agrava con la adhesión de los detergentes (Cevallos, 2005), que a su vez incrementan la concentración de fósforo en el agua residual (Gomes, 2015). Además, son compuestos orgánicos más estables y por su densidad forman una película en la interfaz aire-agua, impidiendo que se desarrollen procesos biológicos normales e inhabilitando el intercambio de gases entre las dos fases, lo cual reduce el oxígeno disuelto, haciendo más lenta la autodepuración de los ríos y fuentes de agua. Estos efluentes con aceiten minerales quemados aportan también con toxinas al agua, como por ejemplo alquibenzenos, naftaleno, tricloretano y metales propios del desgaste del motor como bario, alumnio, plomo, zinc, cromo (Departamento de Medio Ambiente de Aragón, 2007), reduciendo la diversidad microbiana (Nyman, 1999).

Para regular este problema la normativa ambiental nacional en Ecuador prohíbe el desecho en el agua de aceites y grasas en una concentración superior a $70 \mathrm{mg} / \mathrm{L}$ (MAE, 2015). Para el tratamiento de este tipo de aguas residuales las lubricadoras utilizan métodos físicos de tratamiento, como las trampas de grasa (Ramalho, 1996), los cuales aprovechan la propiedad 
inmiscible de los dos líquidos para realizar una separación física entre agua y contaminante. Esto es validado por varias leyes sectoriales, como la ordenanza de Quito, capital del país, (DMQ, 2005). Sin embargo, estos tratamientos no son suficientes para tratar las grasas emulsificadas, ya que no se toma en cuenta los aceites y las grasas que cayeron sobre el agua, y que debido a su alta temperatura formaron una emulsión, aumenta la carga orgánica (Envitech, 2016); por lo que, Reed et. al. (1998) recomienda la construcción de sistemas de flotación de aire disuelto (FAD) o sistemas de ultrafiltración, los cuales serían muy costosos para los propietarios de estos establecimientos.

La ciudad de Ambato, en el año 2010 tuvo una población de 287.282 habitantes (INEC, 2011), para el año 2016 el total de lavadoras y lubricadoras de vehículos registradas era de 57 (GAD Ambato 2016). Este caudal es vertido al sistema de alcantarillado de la ciudad, y posteriormente descargado en su mayoría al río Ambato (Panimbosa, 2018).

Con el propósito de tratar la fase soluble de estos efluentes, y aportar con un método de descontaminación con menores costos que tecnología primaria avanzada, se pretende usar un modelo de lodos activos del tipo lecho suspendido, que consiste en un reactor biológico de mezcla completa, en donde un residuo orgánico presente en el agua es estabilizado por una masa de microorganismos activos; todo esto en un medio de aireación forzada. La configuración de este reactor a escala de laboratorio será por lotes; la aireación y la sedimentador se realizará en un mismo tanque con distintos tiempos de retención (Méndez, Miyashiro, Rojas, Cotrado, \& Carrasco, 2004).

\section{Materiales y métodos}

\section{Agua residual}

Los protocolos de análisis fueron realizados según el Standard Methods (APHA, 1998). Para conocer la calidad del agua de afluente, se realizó un muestreo simple en 61 lubricadoras en la ciudad de Ambato (57 registradas y 4 no registradas), analizando DQO, DBO5; sólidos, nitrógeno y fósforo totales; los puntos de muestreo se encuentran ubicados como lo indica el Figura 1. Con estos datos se obtuvieron los valores de Demanda química de oxígeno (DQO), con la técnica de reflujo cerrado y volumetría (Standard Methods 5220C), la Demanda bioquímica de oxígeno $\left(\mathrm{DBO}_{5}\right)$ con la técnica de incubación en 5 días y uso de oxímetro (Standard Methods 4500), sólidos, nitrógeno y fósforo totales.

Usando estos datos se preparó en laboratorio agua residual representativa a un $95 \%$ de ocurrencia, mediante una emulsión desde aceite quemado para la etapa experimental. 


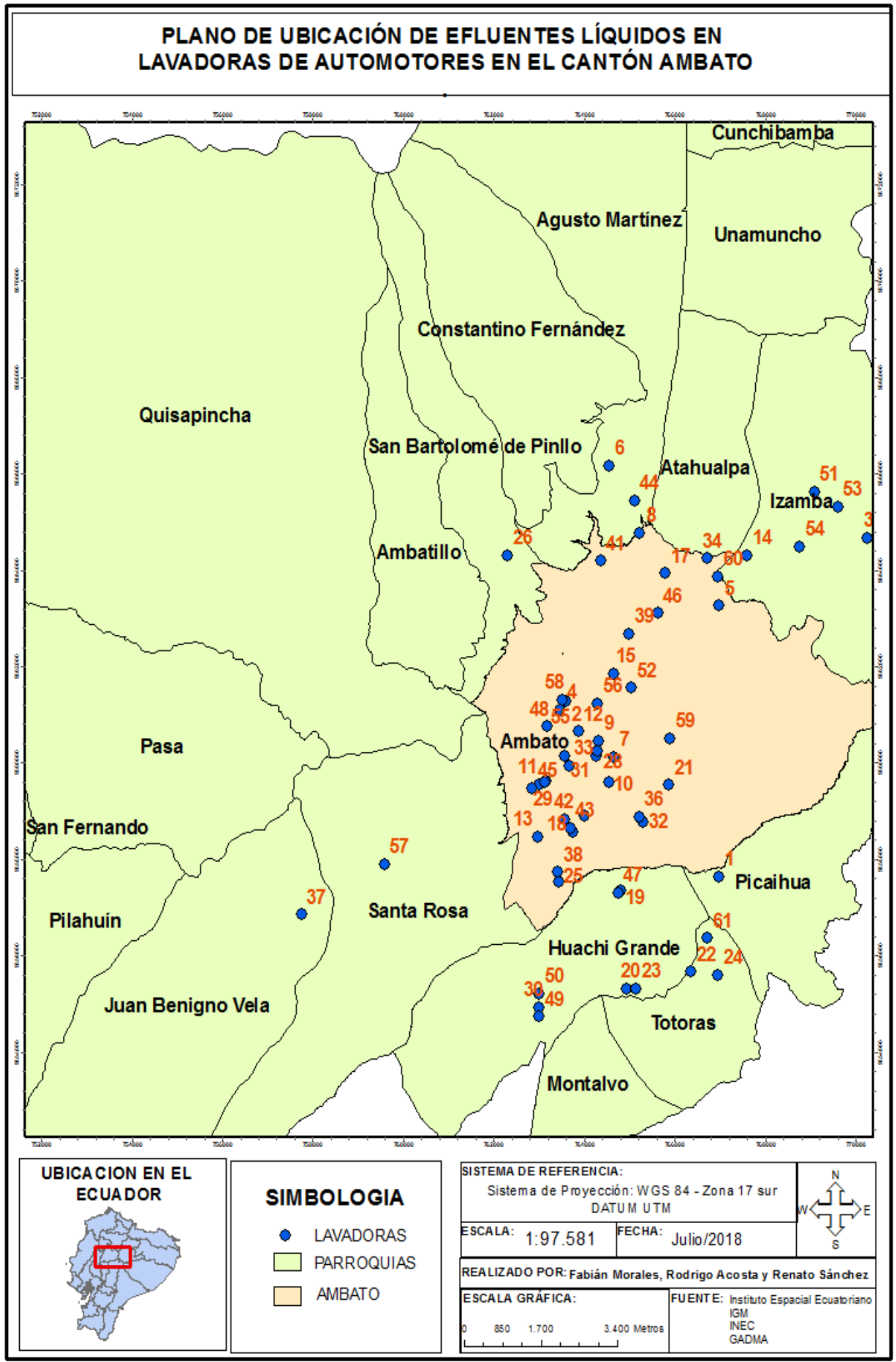

Figura 1. Ubicación de las lavadoras-lubricadoras 


\begin{tabular}{lcllll}
\hline $\begin{array}{l}\text { Parámetros } \\
\text { calidad }\end{array}$ & $\begin{array}{l}\text { de } \\
\text { DQO } \\
(\mathrm{mg} / \mathrm{L})\end{array}$ & $\begin{array}{l}\mathrm{DBO}_{5} \\
(\mathrm{mg} / \mathrm{L})\end{array}$ & $\begin{array}{l}\text { Sólidos } \\
\text { totales } \\
(\mathrm{mg} / \mathrm{L})\end{array}$ & $\begin{array}{l}\text { Nitrógeno } \\
\text { total }(\mathrm{mg} / \mathrm{L})\end{array}$ & $\begin{array}{l}\text { Fósforo total } \\
(\mathrm{mg} / \mathrm{L})\end{array}$ \\
\hline Probabilidad 95\% & 7950 & 3895 & 86792,33 & 1140,34 & 47,52 \\
\hline
\end{tabular}

Tabla 1. Valores de calidad de agua a usarse en la investigación experimental

En la preparación de la emulsión se realizó primero una curva de calibración entre cantidad de aceite colocado y DQO alcanzado. Posteriormente se obtuvo la muestra usando esta curva de calibración, en el cual se obtuvo un valor de DQO en $7930 \mathrm{mg} / \mathrm{L}$, cercano al valor de 95\% de probabilidad; concentración a la cual se realizó la experimentación.

\section{Protocolos de análisis}

Los protocolos de análisis para los parámetros de Demanda química de oxígeno (DQO), Demanda bioquímica de oxígeno $\left(\mathrm{DBO}_{5}\right)$ y sólidos totales, nitrógeno total y fósforo total fueron realizados según el Standard Methods (APHA, 1998).

\section{Proceso de lodos activos de lechos suspendido}

La configuración del reactor de lodos activados que se utiliza experimentalmente es por lotes (Moreno \& Buitrón, 2012); según Romero (2010) para el correcto funcionamiento de un sistema biológico debe existir una relación de carbono, nitrógeno y fósforo de 100/5/1; mientras que en el agua residual existe una relación promedio de 100/29/1.

Debido a que el agua residual que ingresa al tratamiento biológico carece de fósforo, se colocará como lecho suspendido retazos sólidos de caña de azúcar Saccharum officinarum (Chávez, 1999). Si bien, la adición de la Saccharum officinarum en el agua incrementa la carga orgánica en $\mathrm{DQO}$ y $\mathrm{DBO}_{5}$, ésta también mejora la relación $\mathrm{DBO}_{5} /$ nitrógeno/fósforo a niveles a los cuales se puede tratar el efluente mediante tratamiento biológico.

Para la construcción de este reactor se usó policarbonato; las medidas del reactor fueron de 0,30 metros por 0,30 metros por 0,2 metros; para la aireación se usaron cinco aspersores de burbuja fina. Se realizó una cámara de separación de las grasas no emulsionadas en la parte superior del reactor (Oliveira, 2019); este prototipo de lo encuentra en la Figura 2.

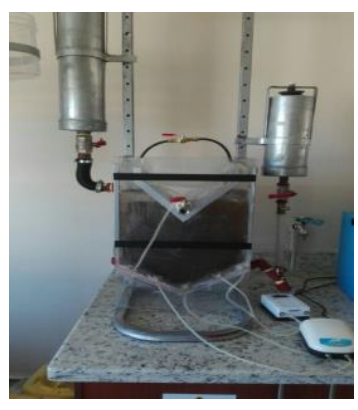


Figura 2. Prototipo usado en la fase experimental

\section{Resultados.}

En la Tabla 2 se muestran los datos de DQO, medidos al inicio y al final de cada tiempo de retención y sólidos sedimentables. La medición de DQO se realizó con un equipo marca Hanna, modelo HI 83224, mediante el método 5220 D (APHA, 1998). Para el cálculo de las tasas de remoción de DQO se procesan los datos como se representa en la Tabla 3; y para la obtención de los coeficientes cinéticos se usaron las Figuras 4 y 5 (Techobanglous, 1995).

\begin{tabular}{|c|c|c|c|c|c|c|}
\hline Experiencia & $\begin{array}{l}\text { DQO } \\
\text { inicial } \\
\text { So, } \mathrm{mg} / \mathrm{L}\end{array}$ & $\begin{array}{l}\text { DQO } \\
\text { final } \\
\mathrm{S}, \mathrm{mg} / \mathrm{L}\end{array}$ & $\begin{array}{l}\text { Volumen } \\
\text { ml }\end{array}$ & $\begin{array}{l}\text { densidad } \\
\text { gr/ml }\end{array}$ & $\begin{array}{l}\theta=\theta c \\
\text { días }\end{array}$ & $\begin{array}{l}X \\
\mathrm{mg} / \mathrm{L}\end{array}$ \\
\hline 1 & 7930 & 6016 & 0,33 & 0,9 & 3 & 297 \\
\hline 2 & 7930 & 6385 & 0,33 & 0,9 & 2 & 297 \\
\hline 3 & 7930 & 6644 & 0,33 & 0,9 & 1 & 297 \\
\hline
\end{tabular}

Tabla 2: Datos tomados en la fase experimental

\begin{tabular}{|c|c|c|c|c|c|c|}
\hline $\begin{array}{l}\text { So-S } \\
\mathrm{mg} / \mathrm{L}\end{array}$ & $\begin{array}{l}\text { XO } \\
(\mathrm{mg} / \mathrm{l}) * \mathrm{~d}\end{array}$ & $\begin{array}{l}\text { XO/So-S } \\
\text { d }\end{array}$ & $\begin{array}{l}\text { 1/So } \\
\mathrm{L} / \mathrm{mg}\end{array}$ & $\begin{array}{l}1 / \theta c \\
d-1\end{array}$ & $\begin{array}{l}\text { So-S/XO } \\
\text { d-1 }\end{array}$ & $\begin{array}{l}1 / \mathrm{S} \\
(\mathrm{mg} / \mathrm{L})-1\end{array}$ \\
\hline 1914 & 891 & 0,466 & $1,26 \mathrm{E}-04$ & 0,33 & 2,148 & 0,00016622 \\
\hline 1545 & 594 & 0,384 & $1,26 \mathrm{E}-04$ & 0,50 & 2,601 & 0,00015662 \\
\hline 1286 & 297 & 0,231 & $1,26 \mathrm{E}-04$ & 1,00 & 4,330 & 0,00015051 \\
\hline
\end{tabular}

Tabla 3: Tasas de remoción de DQO en el reactor de lecho suspendido

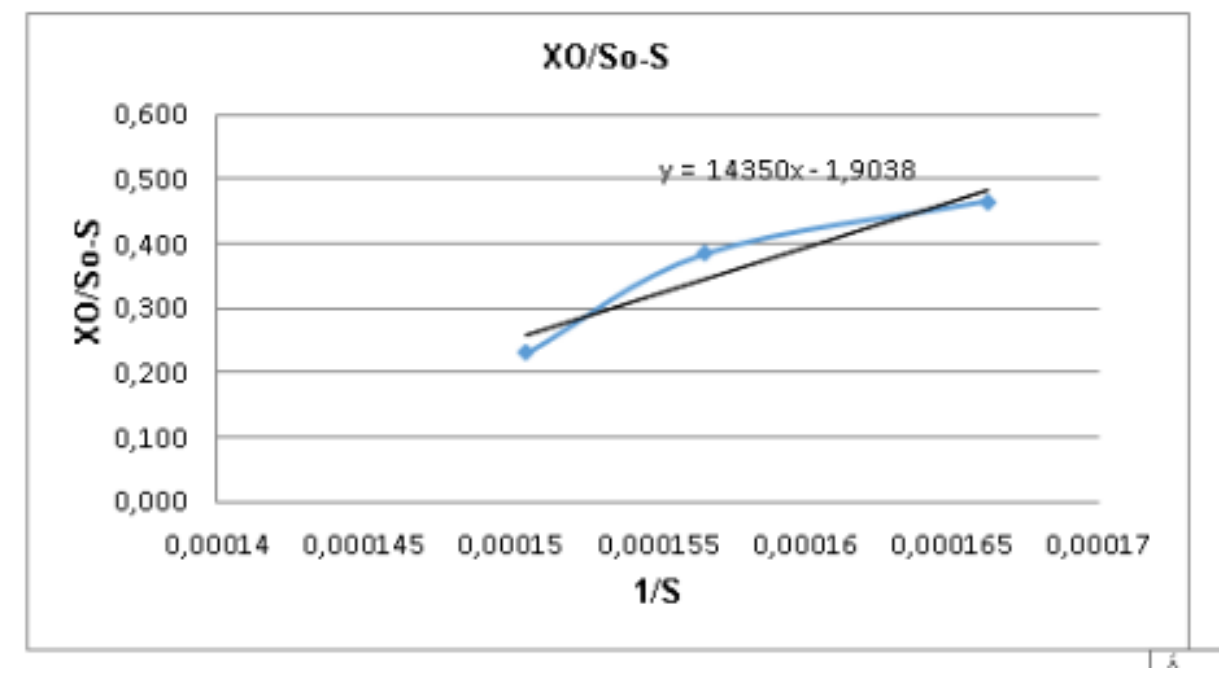

Figura 4: Obtención de los coeficientes cinéticos: Sólidos por carga removida 


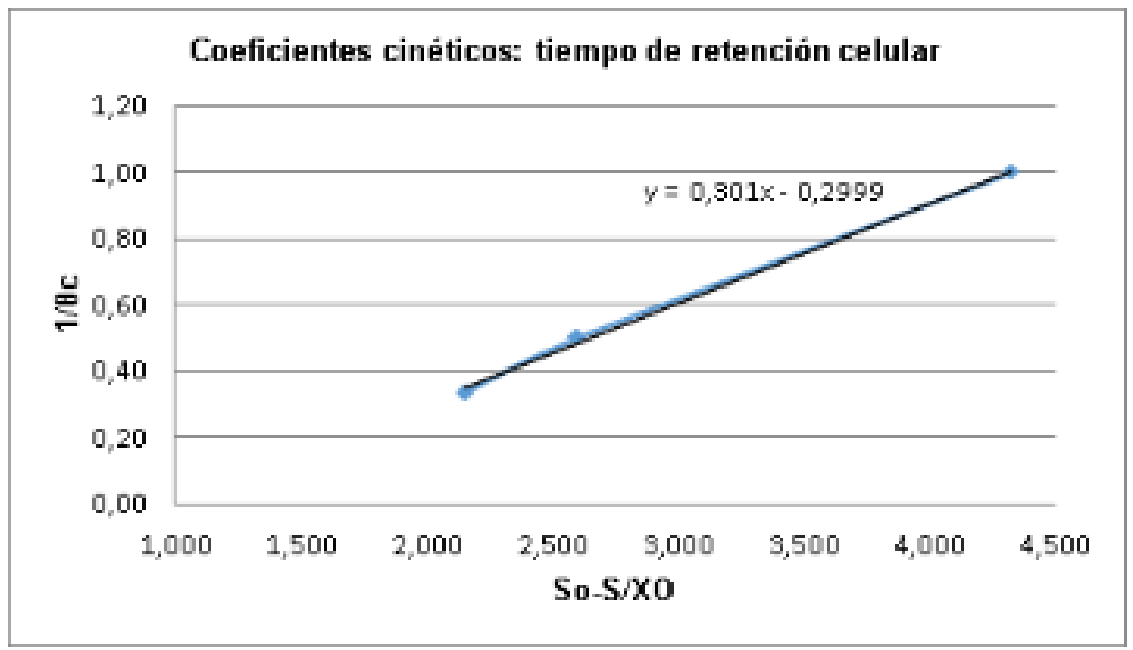

Figura 5: Gráfico para obtención de los coeficientes cinéticos para tiempo de retención celular

Las tablas muestran el grado de descontaminación del agua residual, así como las tasas de remoción del contaminante y con estos datos se puede estimar que el tiempo de descontaminación hasta llegar a norma de $500 \mathrm{mg} / \mathrm{L}$ en DQO es de 16,18 días.

\section{Discusión}

Si bien, la legislación exige la construcción de trampas de grasa en este tipo de actividades económicas, se puede apreciar que parte de estos residuos producen una emulsión en el agua residual, aumentando de esta manera la carga orgánica en los efluentes, que no son separados en las trampas de grasa. Esta carga orgánica puede separarse con sistemas primarios avanzados, los cuales son costosos; pudiéndose reemplazar con sistemas tratamiento con sistemas biológicos; cuyo funcionamiento se demostró en esta investigación.

Varios autores señalan que el tratamiento biológico no resulta eficiente debido a los compuestos tóxicos y poco biodegradables que los aceites minerales pueden tener. En el presente estudio se ha demostrado que un método válido para la remoción de la carga contaminante es el tratamiento de lodos activados de lecho suspendido con soporte de bagazo de caña (Saccharum officinarum), que, si bien los microorganismos tardan en adaptarse al medio, una vez superada esta etapa el tratamiento funciona correctamente.

\section{Conclusiones}

- Es importante tomar en cuenta que mientras la carga contaminante se va reduciendo al mismo tiempo el oxígeno disuelto va aumentando, esto quiere decir que el agua va recuperando características necesarias para que las actividades microbiológicas se vayan desarrollando de mejor manera así también los procesos biológicos y físicos. 
- Aunque hay diversos métodos para la separación de aceites y grasas presentes en aguas residuales es necesario elegir el adecuado para obtener el grado de disociación óptimo que nos permita una remoción completa del contaminante y de esta manera disponer el agua tratada al efluente elegido.

- La contaminación en el rio Ambato ha llegado a niveles alarmantes ya que es el cuerpo de agua que más recibe las aguas residuales tanto de industrias como las domesticas, mediante este tratamiento el agua proveniente de las lubricadoras y lavadoras del cantón ya no sería una carga significativa perjudicial al rio.

\section{Agradecimientos}

Este proyecto es financiado por la Universidad Técnica de Ambato, sin este apoyo no hubiera sido posible llevar a buen fin el mismo.

\section{Referencias Bibliográficas.}

APHA (1998) Standard methods for the examination of water and wastewater. 20a ed. Washington: American Public Health Association. DOI: 10.1016/00489697(94)90332-8.

Aranberri, I., Binks, P., Clint, H., \& Fletcher, I. (2010). Elaboración y caracterización de emulsiones estabilizadas por polimeros y agentes tensioactivos. Revista Iberoamericana de Polímeros.

Cevallos, G. (2005). Estudio de la biodegradación de los detergentes comerciales domesticos de nuestro país.

Chávez, M. (1999). El Nitrogeno, Fósforo Y Potasio En La Caña De Azúcar . Obtenido de https://www.laica.co.cr/biblioteca/servlet/DownloadServlet?c=443\&s=1762\&d=174 4

Debatara, M y Somnath Mukherjee (2011). International Journal of Environmental Science and Development, Vol.2, No.1, February 2011 ISSN: 2010-0264.

Departamento de Medio Ambiente de CCOO- Aragón. (2007). Guía para la reducción del Impacto ambiental de los aceites. Recuperado desde: http://www.aragon.ccoo.es/comunes/recursos/3/doc148742.

Districto Metropolitano de Quito (2005), Ordenanza Sustitutiva del Título V, "Del Medio Ambiente" (Ordenanza No. 146).

Envitech, C. (2016). Tratamiento de emulsiones y aguas residuales aceitosas. Obtenido de https://www.aguasresiduales.info/revista/blog/tratamiento-de-emulsiones-y-aguasresiduales-aceitosas.

Gomes , C. y da Silva W. (2016). Detergents as a Source of Phosphorus in Sewage: the Current Situation in Brazil. W. Water Air Soil Pollut (2016) 227: 14. https://doi.org/10.1007/s11270-015-2700-3. 
Jiménez, S. (2012). Estudio teórico para el control de la contaminación por grasas y aceites generadas por la actividad industrial, doméstica y de servicios. Obtenido de https://tesis.ipn.mx/jspui/bitstream/123456789/18479/1/25-1-16898.pdf

Lan W.U. , G.E. Gang, and W.A.N. Jinbao, Biodegradation of oil wastewater by free and immobilized Yarrowia lipolytica W29, Journal of Environmental Sciences vol. 21, pp. 237-242, 2009.

MAE. (2015). Texto Unificado de Legislación Secundaria : Norma de Calidad Ambiental y de Descarga de Efluentes al Recurso Agua. Libro VI, Anexo 1, Tabla 8.

Márquez, F., \& Pucuna, F. (2015). Análisis de los desechos sólidos y líquidos que generanlas lavadoras de automóviles y su incidencia en el medio ambiente en el cantón milagro.

Méndez, L., Miyashiro, V., Rojas, R., Cotrado, M., \& Carrasco, N. (2004). Tratamiento De Aguas Residuales Mediante Lodos Activados A Escala De Laboratorio. Revista del Instituto de Investigación FIGMMG, 74-83.

Moreno, I., \& Buitrón, G. (2012). Comparación del rendimiento de los reactores por lotes de membrana y convencionales que degradan el 4-clorofenol. Contaminación del aire, agua y suelo, 2083-2091.

Nyman (1999). Effect of Crude Oil and Chemical Additives on Metabolic Activity of Mixed Microbial Populations in Fresh Marsh Soils. Microbial Ecology February 1999, Volume 37, Issue 2, pp 152-162.

Oliveira, V., Alves, J., Basto, L y Pedroso, L. (2019). Esters from frying oil, sewage scum, and domestic fat trap residue for potential use as biodiesel. Renewable Energy Volume 135, May 2019, Pages 945-950.

Panimbosa, R. (2018). Contaminación del rio Ambato. Ambato. Obtenido de https://es.scribd.com/document/391762172/Informe-Ingenieria-Ambientalcontaminacion-Del-Rio-Ambato

Ramalho, R. (1996). Tratamiento de Aguas Residuales. Barcelona: Editorial Revertè S.A.

Reed, Brain E.; Carriere, Patrick; Lin, Wei; Roark, Gary and Viadero, Roger (1998). Oily waste water treatment by ultrafiltration - pilot scale results and full scale design. Practice Periodical of Hazardous, Toxic, and Radioactive Waste Management ; 12(3), pp. $100-107$

Ruiz, E. (1991). Aceites lubricantes para motores a gasolina. Obtenido de http://championchile.cl/wp-content/uploads/2015/04/Curso-de-Lubricacion.pdf

Techobanglous G. B. , and L. Franklin, Wastewater Engineering, 3rd edition, Metcalf and Eddy Inc., 1995. (incluir y dar formato a esta referencia)

Vidales, A., Leos, M., \& Campos, M. (2010). Extracción de Grasas y Aceites en los Efluentes. Conciencia Tecnológica, 40, 29-34.

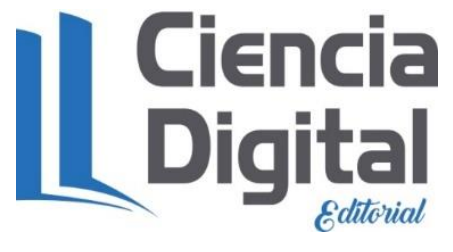




\section{Para citar el artículo indexado.}

Morales F., Sánchez R. \& Acosta R. (2019). Tratamiento de efluentes de lubricadoras y lavadoras de autos mediante un sistema de lodos activados de lechos suspendido a escala de laboratorio. Revista electrónica Ciencia Digital 3(1), 170-179. Recuperado desde: http://cienciadigital.org/revistacienciadigital2/index.php/CienciaDigital/article/view/278/67 $\underline{0}$

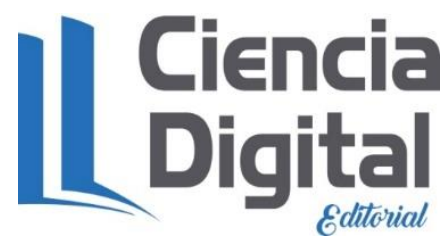

El artículo que se publica es de exclusiva responsabilidad de los autores y no necesariamente reflejan el pensamiento de la Revista Ciencia Digital.

El artículo queda en propiedad de la revista y, por tanto, su publicación parcial y/o total en otro medio tiene que ser autorizado por el director de la Revista Ciencia Digital.
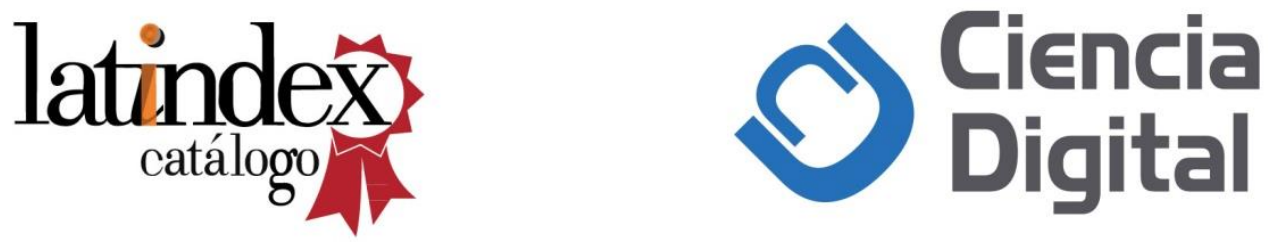